\title{
Threshold Behaviour in Gauge Boson Pair Production at LEP 2
}

\author{
F. Boudjema ${ }^{1}$ and N. Dombey ${ }^{2 *}$ \\ ${ }^{1}$ Laboratoire de Physique Théorique ENSLAPP \\ Chemin de Bellevue, B.P. 110, F-74941 Annecy-le-Vieux, \\ Cedex, France \\ ${ }^{2}$ School of Mathematical \& Physical Sciences, University of \\ Sussex, Falmer, Brighton, Sussex, BN1 9QH, UK
}

September 30, 2018

\begin{abstract}
We discuss the form of the amplitude for gauge boson pair production at or near threshold.We show that in the case of $\mathrm{W}$-pair production at LEP2 near threshold only one anomalous electromagnetic coupling can contribute. This anomalous coupling is $\mathrm{CP}$ violating and contributes to the electric dipole moment of the $W$. Since this coupling is likely to be small, it is important to look for $Z Z \gamma$ couplings in $Z \gamma$ production. These couplings are not suppressed at the $W$ threshold.
\end{abstract}

*SUSX-TH-95/47, Submitted to Physics Letters B 


\section{Introduction}

CERN intends to increase the energy of its electron-positron collider LEP so that $W$-pairs can be produced. The upgraded LEP 2 should demonstrate directly the presence of the non-Abelian gauge coupling of the $Z$ to $W^{+} W^{-}$. It should also show the effects of the magnetic moment and the quadrupole moment of the $W$. At tree-level, these quantities are predicted by the standard electroweak model without any assumptions on its Higgs content but have not yet been measured directly 円. The energy of LEP 2 may eventually be increased to allow the production of $Z$-pairs in addition to $W$-pairs.

Many authors including ourselves have considered alternatives to the standard electroweak model in which additional self-couplings of the gauge particles can occur [1, 2, 3, 国. These new anomalous couplings are often present in the standard model as small radiative corrections, but in alternative models it is commonly speculated that they may be large. For example, the $Z$ should couple to an electromagnetic field through a $C$ - and $P$-violating, but $C P$-conserving, anapole moment of order $\alpha / \pi$ in the standard model[5], but in a composite model this anapole moment may be large, that is to say of order unity [4. Similarly, the $W$ has an electric dipole moment (edm) of order less than $10^{-38} e \mathrm{~cm}^{2}$ ? in the minimal standard model, but in alternative models it may be much larger and if so, this edm may even be the source of the $C P$-violation observed in the $K^{0}-\bar{K}^{0}$ system. Nevertheless, even if such anomalous couplings exist, they are not expected to have any appreciable consequences in measurements at current LEP energies. An important aim of LEP 2 is to search for the presence of these anomalous couplings (and other variations from the predictions of the standard model) in addition to its primary purpose of demonstrating the $W W Z$ non-Abelian coupling and the magnetic and quadrupole moments of the $W$.

In this note we consider the general form of the three vector boson coupling which gives in particular the electromagnetic structure of the $W$ and the $Z$. This allows us to formulate threshold theorems for all the gauge boson pair production processes. In the next section we show that a threshold theorem exists for $e^{+} e^{-} \rightarrow$ $W^{+} W^{-}$which picks out one particular anomalous coupling. In section 3 we discuss $e^{+} e^{-} \rightarrow Z Z$ which is especially simple to analyse on account of the Majorana (self-dual) nature of the $Z$. This property severely limits the form

\footnotetext{
${ }^{1}$ Present limits from the Tevatron are too large to be meaningful

${ }^{2}$ The electric dipole moment of the $W$ in the standard model is known to vanish even at the two-loop order [6]. The bound we quote is from an estimate given in reference [7].
} 
of the $Z Z \gamma$ (or $Z Z Z$ ) interaction and gives a very clean threshold theorem. In section 4 we discuss $e^{+} e^{-} \rightarrow Z \gamma$, which satisfies a threshold theorem but at LEP, not LEP 2 energies. It is therefore especially important to study this process at LEP 2 since the constraints of the threshold theorem no longer apply.

\section{Threshold Theorem for $W^{+} W^{-}$Production}

The general form of the electromagnetic coupling of a spin-one particle of mass $M$ can be written in the form [2]

$$
\begin{aligned}
\Gamma^{\alpha \beta \mu}(q, \bar{q}, k)= & \tilde{f}_{1}(q-\bar{q})^{\mu} g^{\alpha \beta}-\frac{\tilde{f}_{2}}{M^{2}}(q-\bar{q})^{\mu} k^{\alpha} k^{\beta}+\tilde{f}_{3}\left(k^{\alpha} g^{\mu \beta}-k^{\beta} g^{\mu \alpha}\right) \\
& +i \tilde{f}_{4}\left(k^{2}\left(k^{\alpha} g^{\mu \beta}+k^{\beta} g^{\mu \alpha}\right)-2 k^{\alpha} k^{\beta} k^{\mu}\right) \\
& +i \tilde{f}_{5}\left(k^{2} \epsilon^{\alpha \beta \mu \rho}(q-\bar{q})_{\rho}+2 k^{\mu} \epsilon^{\alpha \beta \rho \sigma} q_{\rho} \bar{q}_{\sigma}\right) \\
& -\tilde{f}_{6} \epsilon^{\mu \alpha \beta \rho} k_{\rho}-\frac{\tilde{f}_{7}}{M^{2}}(q-\bar{q})^{\mu} \epsilon^{\alpha \beta \rho \sigma} k_{\rho}(q-\bar{q})_{\sigma}
\end{aligned}
$$

Here, $q$ and $\bar{q}$ are the momenta of the $W^{+}$and $W^{-}$and $k=q+\bar{q}$ is the virtual photon momentum. The $W$ is taken to be on-shell so $q^{2}=\bar{q}^{2}=M^{2}$. Since the electromagnetic current is conserved we have

$$
k_{\mu} \Gamma^{\alpha \beta \mu}=0
$$

No symmetry other than electromagnetic current conservation and Lorentz invariance is assumed in this decomposition.

If we now consider the process $e^{+} \mathrm{e}^{-} \rightarrow W^{+} W^{-}$through the virtual photon exchange term of Figure 1, we can ignore all terms in Eq. (1) proportional to $k^{\mu}$ since the electromagnetic current of the electron is also conserved. Hence we obtain the form of the coupling written down by Hagiwara et al. [2]

$$
\begin{aligned}
\Gamma_{T}^{\alpha \beta \mu}(q, \bar{q}, k)= & f_{1}(q-\bar{q})^{\mu} g^{\alpha \beta}-\frac{f_{2}}{M^{2}}(q-\bar{q})^{\mu} k^{\alpha} k^{\beta}+f_{3}\left(k^{\alpha} g^{\mu \beta}-k^{\beta} g^{\mu \alpha}\right) \\
& +i f_{4}\left(k^{\alpha} g^{\mu \beta}+k^{\beta} g^{\mu \alpha}\right)+i f_{5} \epsilon^{\mu \alpha \beta \rho}(q-\bar{q})_{\rho} \\
& -f_{6} \epsilon^{\mu \alpha \beta \rho} k_{\rho}-\frac{f_{7}}{M^{2}}(q-\bar{q})^{\mu} \epsilon^{\alpha \beta \rho \sigma} k_{\rho}(q-\bar{q})_{\sigma}
\end{aligned}
$$


The seven form factors $f_{i}$ are functions only of $k^{2}: f_{1}(0), f_{2}(0)$ and $f_{3}(0)$ determine the electromagnetic charge, magnetic moment and quadrupole moment of the $W$ and conserve $C, P$ and $T ; f_{6}$ and $f_{7}$ violate $P$ and $T$ but conserve $C$ and hence can lead to an electric dipole moment of the $W$, while $f_{4}$ conserves $P$ but violates $C$ and $T$. The anapole $f_{5}$ conserves $T$ but violates $C$ and $P$. By comparing Equations (1) and (3) we obtain the constraints $f_{4}(0)=f_{5}(0)=0$ imposed by current conservation.

In $W$-pair production at LEP 2 the amplitude to first order in $\alpha$ is given by the Born approximation, that is the neutrino exchange term (Figure 2) which is non-zero at threshold, the virtual photon exchange term of Figure 1, together with a corresponding term in which the virtual photon is replaced by a virtual $Z$. The We $\nu$ coupling is known from $\mu$-decay and $\beta$-decay.

The description of virtual $Z$-exchange is almost identical to that of virtual photon exchange. We now take the $Z W W$ coupling (or weak vertex function of the $W$ ) to be given by $G^{\alpha \beta \mu}$ instead of $\Gamma^{\alpha \beta \mu}$ and we can break up $G^{\alpha \beta \mu}$ into its transverse and longitudinal parts

$$
G^{\alpha \beta \mu}=G_{T}^{\alpha \beta \mu}+G_{L}^{\alpha \beta \mu}
$$

where $G_{L}^{\alpha \beta \mu}$ includes all terms proportional to $k^{\mu}$. Provided that we take $m_{e}=0$, the weak neutral current of the electron- positron system which couples to the $Z$ is conserved. This ensures that the longitudinal part, $G_{L}^{\alpha \beta \mu}$, does not contribute to the amplitude. So we can take the $Z W W$ coupling to be given by $G_{T}^{\alpha \beta \mu}$ and this can be decomposed in exactly the same form as the electromagnetic vertex $\Gamma_{T}^{\alpha \beta \mu}$ in Eq. 1 provided we simply replace the form factors $f_{i}$ by the corresponding form factors $f_{i}^{Z}$. We can also neglect Higgs exchange in the $m_{e}=0$ limit.

At threshold $q=\bar{q}$ in the centre of mass frame and hence $f_{1}, f_{2}$ and $f_{7}$ do not contribute to the virtual photon exchange diagram. Furthermore in this frame $k$, $q$ and $\bar{q}$ are purely timelike while the polarization vectors $\epsilon$ and $\bar{\epsilon}$ of $W^{+}$and $W^{-}$ are purely spacelike. Hence when contracted with $\epsilon^{\alpha}, \bar{\epsilon}^{\beta}, f_{3}$ and $f_{4}$ are coefficients of $k . \epsilon$ and $k . \bar{\epsilon}$ both of which vanish. So only the CP-violating term $f_{6}$ persists at threshold. For $Z$-exchange a similar argument gives $f_{6}^{Z}$ as the sole contribution.

So we end up with the threshold theorem for $W$-pair production at threshold

$$
\frac{\mathrm{d} \sigma\left(e^{+} e^{-} \rightarrow W^{+} W^{-}\right)}{\mathrm{d} \Omega}=\frac{1}{s}\left(\frac{M_{W}^{2} G_{\mu}}{\pi \sqrt{2}}\right)^{2} \beta\left[1+4 \beta \cos \theta \frac{3 c_{W}^{2}-1}{4 c_{W}^{2}-1}+2 s_{W}^{4} F_{6}^{2}+\mathcal{O}\left(\beta^{2}\right)\right]
$$


where we define

$$
\begin{array}{r}
F_{6}^{2}=f_{6}^{2}+\frac{\hat{f}_{6, Z}^{2}}{2}\left(1+\left(1-\frac{1}{2 s_{W}^{2}}\right)^{2}\right)-2 f_{6} \hat{f}_{6, Z}\left(1-\frac{1}{4 s_{W}^{2}}\right) \\
\hat{f}_{6, Z}=\frac{s}{s-M_{Z}^{2}} f_{6}^{Z} \quad ; \quad c_{W}^{2}=1-s_{W}^{2}=\frac{M_{W}^{2}}{M_{Z}^{2}}
\end{array}
$$

Note that the interference term between the $\mathrm{CP}$ violating $Z$ and photon exchange diagrams is suppressed. As expected the leading order terms in $\beta$ (the neutrino exchange t-channel and $\mathrm{CP}$ violating s-channel) give an isotropic angular distribution. The next order term in $\beta$ is solely from the t-channel and leads to an asymmetry that vanishes upon integration.

The corollary of this result is that if the electric dipole moment $f_{6}$ (and any associated weak dipole moment $f_{6}^{Z}$ ) of $W$ is too small to be measurable, then the standard model dominates in $W$-pair production near threshold as the other $W W \gamma$ and $W W Z$ couplings all enter into the cross-section with factors of at least $\beta$, where $\beta$ is the velocity of the $W$. This result just follows from kinematics together with electromagnetic (and weak) current conservation, just as it does in classic low energy theorems. [8] It requires no assumptions on the underlying gauge structure of the theory. Furthermore as the We $\nu$ coupling is taken from its value as observed in $\beta$-decay the Born approximation includes the radiative corrections associated with the running of the fine structure constant.

It can be expected that an electric dipole moment $f_{6}$ (and any associated weak dipole moment $f_{6}^{Z}$ ) of the $W$ would contribute to the neutron electric dipole moment. The experimental limits on the neutron edm allow bounds to be set on the $W$ edm. These bounds, however, are not rigorous [9] and LEP 2 can be expected to provide a more substantive result.

In this discussion we have not considered the radiative corrections to the process, in particular the initial state radiation effects which give the dominant contribution (see the treatment by Bardin et al [10] and Berends, Pittau and Kleiss [1]]). Nor have we considered the finite width effects which can be substantial in the threshold region. These may be treated according to the prescription of Fadin, Khoze and Martin. [12]

\section{Threshold Theorem for $e^{+} e^{-} \rightarrow Z Z$}

Extensive studies have been devoted to anomalous $W W \gamma$ and $W W Z$ couplings but rather less attention has been focussed on the anomalous $Z Z \gamma$ and $Z Z Z$ couplings. There may be three reasons for this: (a) within standard electroweak 
theory the $W W$ cross section is an order of magnitude larger than the $Z Z$ cross section; (b) at LEP 2 there is not expected to be sufficient energy initially to produce $Z$-pairs and (c) $W W Z$ and $W W \gamma$ couplings appear at tree level in standard electroweak theory $Z Z \gamma$ and $Z Z Z$ couplings are absent.

Nevertheless, from the purely phenomenological point of view that we have adopted here the $Z Z \gamma$ and $Z Z Z$ coupling are particularly easy to analyse. This is because the $Z$ is a Majorana particle 13 (i.e. it is its own antiparticle) and hence, using the same notation that we used in the previous section, the $Z Z \gamma$ on-shell coupling must be symmetric under interchange of the two $Z \mathrm{~s}$; that is to say that

$$
\Gamma^{\alpha \beta \mu}\left(q, \bar{q}, k^{2}\right)=\Gamma^{\beta \alpha \mu}\left(\bar{q}, q, k^{2}\right) .
$$

By inspection it is easily seen that only the anapole couplings $\tilde{f}_{4}\left(k^{2}\right)$ and $\tilde{f}_{5}\left(k^{2}\right)$ satisfy this criterion. Imposing CP conservation only $\tilde{f}_{5}\left(k^{2}\right)$ survives. Likewise, for $Z Z Z$ coupling, the corresponding weak anapole moment $\tilde{f}_{5}^{Z}$ is the only term, provided that the first two $Z \mathrm{~s}$ are on-shell and the third off-shell.

So the threshold theorem for $Z$-pair production follows simply: the crosssection at threshold is given by the standard model since the $Z$ cannot possess an electric dipole moment. At higher energies the anapole term which is pure $P$ wave with a $\left(1+\cos ^{2} \theta\right)$ angular distribution should play a role. Note that whereas in $W$-pair production above threshold up to six anomalous couplings may be present outside the threshold region, in $Z$-pair production there is only the anapole. The anapole couples a longitudinal $Z$ to a transverse $Z$, so the signal for the presence of the anapole would arise in the $\sigma_{T L}$ cross-section. The unique $\mathrm{CP}$ conserving $Z Z \gamma$ operator which contributes to $Z Z$ production is given by

$$
\mathcal{L}_{A}=\frac{e \kappa_{A}}{\Lambda_{A}^{2}} F_{\mu \nu} \partial^{\mu}\left(\tilde{Z}^{\alpha \nu} Z_{\alpha}\right)
$$

In momentum space this operator is proportional to the invariant mass $k^{2}$ of the photon (see Eq. 1) which means that in $e^{+} e^{-}$processes this leads to a contact interaction [13]. The $Z Z Z$ vertex on account of Bose symmetry is of the same Lorentz structure as the anapole with the $\left(k^{2}\right)$ term replaced by $k^{2}-M_{Z}^{2}$ : Again it also leads to a contact interaction. For these reasons unless the initial beams are polarised we cannot distinguish between a $Z Z Z$ and $Z Z \gamma$ anomalous coupling in $Z Z$ production. For illustration we show the contribution of the electromagnetic operator to the differential $Z Z$ cross section

$$
\frac{d \sigma_{T L}}{d \cos \theta}=\frac{\pi \alpha^{2}\left(M_{Z}^{2}\right)}{M_{Z}^{2}} \beta\left\{G_{Z Z} \frac{4 \cos ^{2} \theta+\left(2-\gamma \sin ^{2} \theta\right)^{2}}{\left(4+\gamma^{2} \beta^{2} \sin ^{2} \theta\right)}+\left(\frac{k_{A} M_{Z}^{2}}{4 \Lambda_{A}^{2}}\right)^{2} \gamma^{2} \beta^{4}\left(1+\cos ^{2} \theta\right)\right\}
$$


where $\beta^{2}=1-4 M_{Z}^{2} / s, \gamma=s / M_{Z}^{2}$ and $G_{Z Z}=G_{Z \gamma}^{2}+4 X_{Z \gamma}^{2}$. The quantities

$$
G_{Z \gamma}=\frac{\left(1-4 s_{W}^{2}\right)^{2}+1}{16 s_{W}^{2} c_{W}^{2}}
$$

and

$$
X_{Z \gamma}=-\frac{\left(1-4 s_{W}^{2}\right)}{16 s_{W}^{2} c_{W}^{2}}
$$

will occur in $e^{+} e^{-} \rightarrow Z \gamma$.

Note that the anomalous term is strongly suppressed by a function $\beta^{4}$ compared to the standard model terms where $\beta$ is the $Z$ velocity. So energies well above the planned LEP 2 energies would be necessary to investigate this coupling. Nevertheless it is perhaps worth noting that at a future electron-positron collider of energy $500 \mathrm{GeV}$ or more, it should be relatively easy to disentangle the standard model terms from the anomalous anapole term. This would be accomplished by a cut in scattering angle about the forward direction which would severely restrict those events arisng from the standard model while hardly affecting the anomalous terms.

\section{Threshold Theorem for $e^{+} e^{-} \rightarrow Z \gamma$}

We showed some years ago [14] (see also [15]) that the threshold amplitude for $e^{+} e^{-} \rightarrow Z \gamma$ was determined by the $Z e^{+} e^{-}$coupling constant including one-loop radiative corrections. This is simply a statement that the threshold energy for this process is the $Z$ mass and therefore soft-photons have to be treated according to quantum electrodynamics in the usual way. So near threshold, the standard model term for this process which correctly contains the threshold amplitude must dominate over all anomalous terms.

The $Z$-exchange diagram for the process $e^{+} e^{-} \rightarrow Z \gamma$ where one $Z$ is on-shell and one is off-shell and the photon is real, involves a coupling of electric dipole transition type that vanishes when both Z's become real. The CP conserving operator that parametrizes this transition can be written as

$$
\mathcal{L}_{E}=\frac{e \kappa_{E}}{\Lambda_{E}^{2}} Z_{\mu \nu} \partial^{\mu}\left(\tilde{F}^{\alpha \nu} Z_{\alpha}\right) \quad \tilde{F}^{\mu \nu}=\frac{1}{2} \epsilon^{\mu \nu \lambda \rho} F_{\lambda \rho}
$$

where $F^{\mu \nu}$ is the electromagnetic tensor, $\Lambda_{E}$ is the scale associated to this operator and $\kappa_{E}$ characterises the strength of the (EDT) coupling. It is useful to introduce the dimensionless parameter $k_{E}=\kappa_{E} M_{Z}^{2} / \Lambda_{E}^{2}$

One can already set a bound on this operator from the LEP data since it contributes to the radiative decay $Z \rightarrow f \bar{f} \gamma$. We have: 


$$
B r^{(E D T)}=\frac{\Gamma^{(E D T)}(Z \rightarrow f \bar{f} \gamma)}{\Gamma_{S M}(Z \rightarrow f \bar{f})}=\frac{\alpha}{80 \pi} k_{E}^{2}
$$

The best limit is from the search of single (energetic) isolated photons associated with $\nu \bar{\nu} \gamma$ production. The L3 Collaboration [16] gives the limit $\left|k_{E}\right| \leq 0.80 @ 95 \%$ C.L. In fact this limit corresponds to all events collected for centre-of-mass energies $88.56<\sqrt{s}<93.75 \mathrm{GeV}$. This is not as good a limit as one might hope to get due to the $\mathrm{P}$-wave nature of the transition which makes the photon prefer to have as large an energy as possible, but around the $Z$ peak this is offest by the fact that this configuration forces the intermediate $\mathrm{Z}$ to be far from resonance.

Therefore, in order to look for this anomalous coupling, it is necessary as in $e^{+} e^{-} \rightarrow Z Z$ to be well above threshold. It turns out that while the cross section for the production of a transverse $Z$ in the standard model term is strongly peaked in the forward/backward directions, the cross section for the production of a longitudinal $Z$ has an isotropic angular distribution. So including the anomalous transition it is easy to show that

$\frac{d \sigma_{L}}{d \cos \theta}=\frac{4 \pi \alpha\left(M_{Z}^{2}\right)}{s} G_{Z \gamma} \frac{1}{\beta \gamma}\left\{\left[1+k_{E} \gamma^{2} \beta^{2} \frac{X_{Z \gamma}}{2 G_{Z \gamma}}+\left(\frac{k_{E}}{8}\right)^{2} \gamma^{4} \beta^{4}\right]+\left(\frac{k_{E}}{8}\right)^{2} \beta^{4} \gamma^{4} \cos ^{2} \theta\right\}$

where now $\beta=1-M_{Z}^{2} / s, \gamma=s / M_{Z}^{2}$, and $G_{Z \gamma} X_{Z \gamma}$ were defined above.

Note that again the anomalous term is suppressed near threshold by a factor $\beta^{4}$.

The importance of $e^{+} e^{-} \rightarrow Z \gamma$ compared to the first two processes is that at foreseeable LEP 2 energies, this process is always well above threshold while the first two processes we have discussed are likely to be still in the threshold region. Hence while it will be difficult to detect any anomalous couplings in $\mathrm{e}^{+} e^{-} \rightarrow W W$ at LEP 2 if CP-violating couplings are small and if LEP 2 is operated at or very close to threshold, and essentially impossible to detect anomalous couplings of the $Z$ in $e^{+} e^{-} \rightarrow Z Z$; there should be no difficulty in searching for anomalous couplings of the $Z$ in $e^{+} e^{-} \rightarrow Z \gamma$.

\section{Conclusions}

We have shown that if $\mathrm{CP}$ is nearly conserved, then it will be difficult to measure deviations from the standard model in $W$-pair production at LEP2. The likelihood of a successful measurement of any deviation increases substantially with the energy of the machine. It is therefore essential to work at the highest energies possible. In some ways this result is reminiscent of that of de Rujula et al. [17], who state that the standard model must be satisfied in the LEP2 energy region. 
Our result differs from theirs, however, in several ways: first their result makes no distinction between threshold and non-threshold energies; second our emphasis is on $\mathrm{CP}$-conservation and not $\mathrm{SU}(2) \mathrm{xU}(1)$ local gauge invariance, and third if $\mathrm{CP}$ is badly broken in the gauge boson sector then a clear discrepancy from the standard model will be observed. Finally we stress again the importance of looking for at $Z \gamma$ production at LEP2 since this process is well above threshold.

\section{References}

[1] K.J.F. Gaemers and G.J. Gounaris, Z. Phys. C 1 (1979) 259.

[2] K. Hagiwara, R.D. Peccei, D. Zeppenfeld and K. Hikasa, Nucl. Phys.B282 253 (1987).

[3] P. Méry, M. Perrotet and F.M. Renard, Z. Phys. C36 249 (1987); idem C38 579 (1988).

[4] F. Boudjema and N. Dombey, Z. Phys. C35 449 (1987).

[5] A. Barroso, F. Boudjema, N. Dombey and J. Cole, Z. Phys. C28 149 (1985).

[6] I.B. Khriplovich and M.E. Pospelov, YFZ 53 1030; Sov. J. Nucl. Phys. 53 638 (1991).

[7] D. Chang, W.Y. Keung and J.L. Liu, Nucl. Phys. B355 295 (1991).

[8] F.E. Low, Phys. Rev. 110974 (1958).

[9] F. Boudjema, K. Hagiwara, C. Hamzaoui and K. Numata, Phys. Rev D43 (1991) 2223. F. Boudjema, C.P. Burgess, C. Hamzaoui and J.A. Robinson, Phys. Rev. D43 3683 (1991).

[10] D. Bardin, M. Bilenkii, A. Olchevskii and T. Riemann, Phys. Lett. B308, 403 (1993).

[11] F.A. Berends, R. Pittau and R. Kleiss, Nucl. Phys. B226 (1994) 344.

[12] V.S. Fadin, V.A. Khoze and A.D. Martin, Phys. Lett. B311, 311 (1993).

[13] F. Boudjema and C. Hamzaoui, Phys. Rev D43 (1991) 3748.

[14] F. Boudjema, N. Dombey and J. Cole, Phys. Lett. B177 (1986) 197.

[15] F. Berends, G.J.H. Burgers and W.L. Neerven, Phys. Lett. B177 (1986) 191.

[16] L3 Collaboration, Phys. Lett. B297 (1992) 469. 
[17] A. de Rújula, M.B. Gavela, P. Hernandez and E. Massó, Nucl. Phys. B384 (1992) 3.

\section{Figure Captions}

Figure 1: $e^{+} e^{-} \rightarrow W^{+} W^{-}$via a virtual photon.

Figure 2: $e^{+} e^{-} \rightarrow W^{+} W^{-}$by means of neutrino exchange. 
This figure "fig1-1.png" is available in "png" format from: http://arxiv.org/ps/hep-ph/9503243v2 


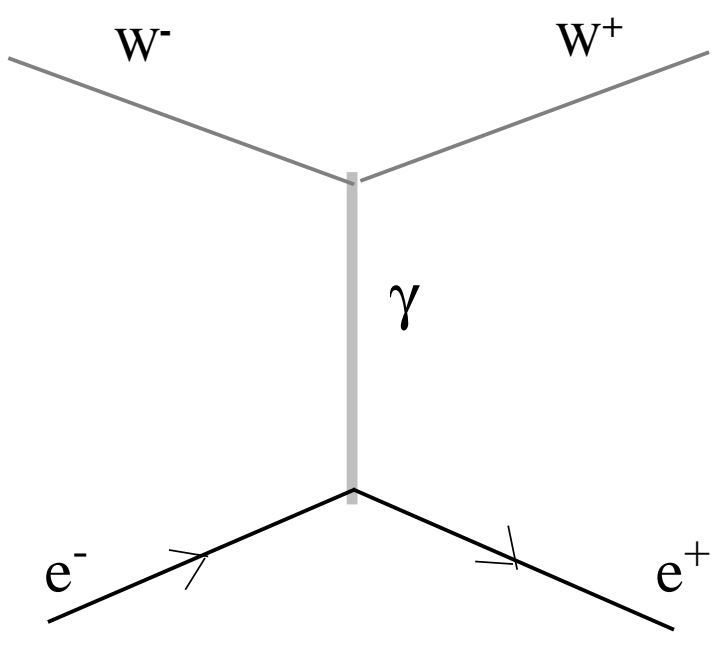

Figure 1

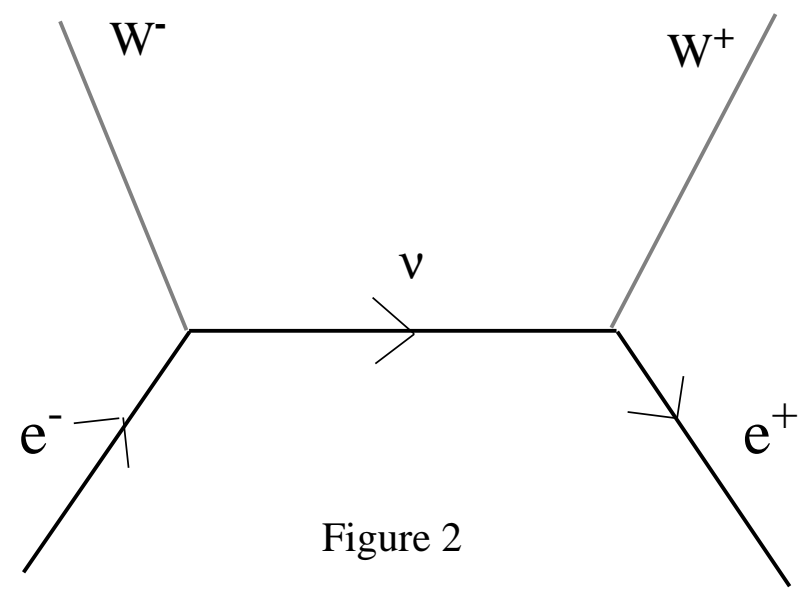

